\title{
Excelência no desporto: Para uma compreensão da "arquitectura" psicológica dos atletas de elite
}

\author{
Excellence in sport: Towards an understanding of the psychological \\ "architecture" of elite athletes
}

\author{
D.S. Matos, J.F.A. Cruz, L.S. Almeida
}

RESUMO

Este estudo teve como objectivo apresentar uma síntese da literatura em torno da excelência, centrando-se no contexto desportivo. Deste modo, procurou-se apresentar as principais abordagens ao estudo da excelência e explorar os factores importantes no seu desenvolvimento e manutenção, considerando um conjunto de dimensões de carácter social e contextual e outras de carácter pessoal. Entre estas últimas, colocou-se especial ênfase nas características e competências psicológicas, de natureza motivacional, afectiva e emocional, numa tentativa de desenhar a "arquitectura" psicológica dos atletas de elite. A caracterização destes atletas, na base da literatura, inclui alguns factores e estratégias consensuais, nomeadamente, os elevados níveis de motivação, comprometimento, concentração e auto-confiança, assim como o recurso a estratégias adaptativas de coping, autoregulação, formulação de objectivos e visualização mental. Ainda que estes resultados demonstrem alguma congruência ao nível da investigação nesta área, ficam por "resolver" as questões relativas ao peso ou importância que cada um destes factores assume ou de que forma é que se combinam para "produzir" desempenhos excelentes.

Palavras-chave: excelência, desporto, alto rendimento, atletas de elite, arquitectura psicológica

ABSTRACT

The purpose of this study was to provide a brief overview of the literature around the field of excellence, focused on the sport's context. Thus, the main approaches to the study of excellence were presented and then some important factors in its development and maintenance were sought considering a set of social and personal dimensions. Among the latter, the focus of attention was on the athlete's psychological, motivational, affective, and emotional characteristics and skills, in an attempt to design the elite athletes' psychological "architecture". The characterization of these athletes included a number of consensual factors and strategies, such as high levels of motivation, commitment, concentration, and self-confidence, as well as the use of adaptive coping strategies, selfregulation, goal-setting and mental imagery. Although these results show some consistency in terms of research, it is still unsolved the issue of the weight and importance of each one of these factors and how such factors are combined in order to "produce" excellent performances.

Keywords: excellence, sport, high performance, elite athletes, psychological architecture

Submetido: 05.10.2010 | Aceite: 08.12.2010

Daniela S. Matos. Bolseira da FCT, Universidade do Minho, Portugal.

José Fernando A. Cruz. Escola de Psicologia, Universidade do Minho, Portugal.

Leandro S. Almeida. Instituto de Educação, Universidade do Minho, Portugal.

Endereço para correspondência: José Fernando Azevedo Cruz, Escola de Psicologia - Centro de Investigação em

Psicologia, Universidade do Minho, Campus de Gualtar, 4710-057 Braga, Portugal.

E-mail: jcruz@psi.uminho.pt 
$\mathrm{Na}$ última década, a necessidade de compreender os desempenhos excelentes no desporto suscitou o interesse da Psicologia e das Ciências do Desporto, tornando-se num tópico central de investigação na comunidade científica. No entanto, apesar da diversidade de estudos, muitas são ainda as dúvidas relacionadas com o conceito de excelência, começando pela sua (in)definição. Neste sentido, coexistem vários termos que lhe estão directa ou indirectamente associados, tais como, expertise, eminence, "expert performance", alto rendimento, elite, mestria e talento, entre outros. Todos estes termos têm implícita a ideia de uma performance elevada e consistente num domínio específico. A par desta dificuldade conceptual, está a multiplicidade de teorias compreensivas da excelência e a diversidade de factores associados, tornando assim evidente a complexidade inerente à sua operacionalização.

Quando reflectimos sobre a psicologia da excelência aplicada a contextos desportivos, atletas como Tiger Woods, Venus Williams, Roger Federer, Yelena Isinbayeva, Cristiano Ronaldo, Ian Thorpe, Sebastien Loeb ou Usain Bolt, surgem-nos, quase de forma automática, devido aos seus desempenhos excepcionais. Também inevitavelmente nos surgem questões como: Quais as características ou competências que tornam estes atletas extraordinários? O que os distingue dos restantes? Como é que desenvolveram essas competências? A resposta a estas questões tem despertado o interesse generalizado dos investigadores na área, daí resultando uma procura sistemática da "arquitectura" psicológica dos atletas de elite.

Deste modo, os objectivos do presente estudo consistiram em: a) apresentar uma breve panorâmica das principais abordagens teóricas ao estudo da excelência em contextos de realização; b) explorar os factores associados à excelência em contextos desportivos; c) identificar, descrever e analisar as características e competências psicológicas associadas ao sucesso dos atletas, e como as mesmas se desenvolvem; e d) analisar a importância dos processos emocionais e afectivos, bem como das estratégias de coping no sucesso desportivo.

\section{DESENVOLVIMENTO}

\section{Abordagens teóricas ao estudo da excelência}

Em termos históricos, Galton (1869) é apontado como o primeiro investigador a estudar a excelência em diferentes domínios e áreas de realização. Desde então, aumentaram os estudos em torno do "fenómeno" e emergiram vários modelos teóricos para o seu enquadramento. Entre eles, destacam-se as abordagens da sobredotação, em que a excelência se associa a conceitos como habilidades inatas, talentos e elevadas competências intelectuais; as abordagens da expertise, onde se destacam conceitos como rendimento superior, peak performance, rendimento perito ou especializado, desempenho excepcional; e, finalmente, as abordagens da sabedoria, em que a excelência se encontra mais associada à experiência de vida (pessoal e profissional) e ao compromisso com os valores e a própria sociedade.

Quando nos centramos na perspectiva da sobredotação, temos inevitavelmente de mencionar os trabalhos pioneiros de Terman (1925), para quem o indivíduo sobredotado deveria apresentar um QI igual ou superior a 145. Esta leitura da sobredotação confinada ao QI permaneceu no tempo, e só a partir dos anos 60 do século passado outras variáveis psicológicas foram consideradas. Nesta altura, a própria sobredotação deixa de ser entendida como um "estado" e, antes, como um processo de desenvolvimento, para o qual convergem variáveis de natureza pessoal e contextual. A título de exemplo, Gagné (2004), no seu Modelo Diferenciado de Sobredotação e Talento (DGMT), considera que as habilidades naturais se traduzem em talento ou excelência numa determinada área quando sujeitas a processos de aprendizagem e de prática, intervindo aí catalisadores de natureza intrapessoal, ambiental e factores de sorte ou oportunidades. Refira-se, também, o trabalho 
desenvolvido por Sternberg (1998, 2001, 2005) onde a excelência é entendida como interacção de competências cognitivas e metacognitivas, competências de aprendizagem e conhecimento, motivação e contexto de realização, no quadro do modelo WICS (wisdom, intelligence, creativity, synthesized) que entende a sobredotação como síntese de sabedoria, inteligência e criatividade. Corroborando esta perspectiva, importa alargar o conceito de sobredotação a outras dimensões de capacidade e desempenho, para além do domínio estritamente cognitivo (Almeida \& Oliveira, 2000).

Por sua vez, as abordagens da expertise/ rendimento superior constituem outra forma de compreender o estudo da excelência, "substituindo" a importância dada pela perspectiva anterior às habilidades naturais por um maior destaque ou papel à experiência e à prática deliberada. De Groot (1946) iniciou esta perspectiva nos seus trabalhos, comparando o desempenho de jogadores de xadrez experts e novatos. Estes estudos continuaram com Simon e Chase (1973), enfatizando o treino nos processos cognitivos para a excelência. Mais recentemente, Ericsson e colaboradores (Ericsson \& Charness, 1994; Ericsson, Krampe, \& Tesch-Römer, 1993; Ericsson \& Lehmann, 1996; Ericsson \& Ward, 2007; Williams \& Ericsson, 2005) desenvolveram uma nova abordagem da excelência, também conhecida como Abordagem ao Rendimento Superior (ARS), salientando a importância decisiva da prática estruturada, sistemática e deliberada.

Uma última abordagem, mais recente na literatura, e desenvolvida na sequência do "movimento" da Psicologia Positiva, é a perspectiva da sabedoria associada aos estudos de Baltes e colaboradores (Baltes \& Kunzmann, 2004; Kunzmann \& Baltes, 2005; Staudinger, Maciel, Smith, \& Baltes, 1998). Segundo estes autores, entende-se por sabedoria o "conhecimento sábio sobre as questões fundamentais da vida" (Baltes \& Staudinger, 2000, p. 122), ou seja, sobre assuntos importantes e, geralmente, contro- versos. Mais recentemente, Kunzmann e Baltes (2003) desenvolveram uma investigação com adultos, concluindo que para além do "conhecimento", a sabedoria estava associada a outras "forças" humanas, nomeadamente a um elevado envolvimento afectivo, integridade pessoal, conhecimento da vida e valores centrados no bem-comum. Estes autores vêm, assim, salientar a importância de três dimensões psicológicas: afectividade, motivação e relacionamento interpessoal.

Em suma, à semelhança de alguma investigação nacional em torno da excelência em diferentes contextos de realização (e.g., Araújo, Cruz, \& Almeida, 2007, 2009; GarciaSantos, Almeida, Werlang, \& Veloso, 2010; Monteiro, Castro, Almeida, \& Cruz, 2009) têm sido notórias as dificuldades inerentes aos estudos nesta área, destacando-se a necessidade de ponderação de diferentes abordagens e dos múltiplos factores que consideram. De qualquer modo, enquanto a perspectiva da sobredotação aparece mais utilizada na análise do desempenho escolar na infância e adolescência, a abordagem do rendimento superior está mais dirigida e centrada no estudo da performance ou realização profissional em jovens-adultos e adultos. Por sua vez, a abordagem da sabedoria parece mais investigada em adultos numa fase de maior integração de conhecimentos e destrezas, colocando a tónica no bem comum.

\section{A excelência em contextos desportivos}

No desporto, assim como noutros contextos de realização (e.g., ciência, música ou artes), alcançar a excelência torna-se o principal objectivo de muitos indivíduos ainda que, só uma "pequena parte", consiga realmente alcançar e manter-se "no topo" (Gagné, 2007). Como consequência, o estudo da excelência ganha crescente relevância na Psicologia e nas Ciências do Desporto, procurando perceber as características e competências dos atletas excelentes, o que os distingue dos restantes atletas, ou como iniciam, desenvolvem e mantêm essas características e competências. 
Uma primeira análise ao estudo da excelência neste contexto específico, revela algumas particularidades que não se fazem necessariamente "sentir" noutros contextos de realização (Thomas \& Thomas, 1994). Assim, a título meramente ilustrativo, enquanto em muitas outras áreas a velocidade na tomada de decisão não é necessariamente essencial para se ser bem sucedido, no caso do desporto, pelo contrário, o sucesso implica processos de tomada de decisões extremamente rápidos e precisos. Outra particularidade, por exemplo, relaciona-se com a possibilidade de não coincidência entre o conhecimento e o desempenho. $\mathrm{O}$ atleta pode saber exactamente como e quando fazer um determinado movimento, mas simplesmente não é capaz de o executar com êxito. Ainda, contrariamente ao que acontece noutros domínios, identificar um atleta excelente na maioria das modalidades não é, "a priori", uma tarefa tão difícil e complexa, pois geralmente em cada uma delas os atletas são classificados através de características e rendimentos objectivos, recorrendo a medidas do tipo "gold standard" (Ericsson, 1996).

Um "olhar" sobre a investigação actualmente existente em torno da excelência no desporto permite-nos perceber que esta se vai repartindo por estudos que se centram mais nos aspectos sociais e contextuais, enquanto outros estão dirigidos para factores pessoais ou de personalidade. Com efeito, as perspectivas sociais e contextuais começam desde muito cedo a ter um lugar "cativo" no estudo da excelência no desporto. Embora não centrado exclusivamente no contexto desportivo, Bloom (1985) foi pioneiro na investigação também nesta área, ao analisar longitudinalmente as carreiras profissionais de jovens talentosos (e.g., músicos, atletas, cientistas e artistas) e ao destacar a importância de determinadas pessoas (influentes e decisivas) nas suas carreiras. Referimo-nos aqui a "outros significativos" como a família, treinadores, professores, amigos e outros agentes que assumem um papel de destaque no percurso dos atletas, fornecendo-lhes apoio social, emocional e/ou instrumental (e.g., económico ou logístico). A importância do apoio social no desenvolvimento de talento em atletas de sucesso é bem ilustrada em estudos recentes, com atletas bem sucedidos, de ambos os sexos e em diferentes modalidades (e.g., Holt \& Dunn, 2004; Morgan \& Giacobbi, 2006). Além da relevância do apoio social positivo por parte das pessoas significativas, outros estudos fazem referência à coesão e coordenação das equipas, à eficácia colectiva e à relação e comunicação eficaz entre treinador e atletas no "caminho para a excelência" (e.g., Gould, Guinan, Greenleaf, Medbery, \& Peterson, 1999; Salas, Cannon-Bowers, \& Johnston, 1997).

Relativamente aos factores de natureza mais individual e pessoal, vários estudos salientam a prática deliberada para um desempenho excepcional no desporto (e.g., Baker \& Horton, 2004; Ericsson \& Charness, 1994; Ericsson et al., 1993; Ericsson \& Lehmann, 1996; Ward, Hodges, Starkes, \& Williams, 2007; Williams \& Ericsson, 2005). No seu estudo clássico sobre a expertise no xadrez, Simon e Chase (1973) sugerem pelo menos 10 anos de intensa preparação, sublinhando, pela primeira vez, a importância decisiva da "regra dos 10 anos" de prática necessária para atingir desempenhos e rendimentos excepcionais em termos internacionais. Esta regra, suportada por dados de diferentes domínios, constitui uma premissa fundamental da chamada "nova ciência do rendimento expert e excepcional" (Ericsson \& Ward, 2007), correspondendo a expertise a "um rendimento superior consistente num conjunto específico de tarefas representativas de um domínio" (Ericsson \& Lehmannn, 1996, p. 277). Para alcançar este nível de rendimento é necessária uma intensa preparação, designada por prática deliberada, que se traduz num "treino individualizado, preparado por um treinador ou professor para aumentar aspectos específicos do desempenho de um indivíduo, através de repetição e 
refinamentos sucessivos" (Ericsson \& Lehmannn, 1996, p. 278). Importa explicitar que a prática deliberada possui algumas especificidades que a tornam diferente do mero treino ou da experiência. Ou seja, esta inclui as actividades que são intencionalmente desenhadas para promover o nível de desempenho actual, é altamente estruturada, implica elevada concentração, é exigente em termos de tempo e energia, não é inerentemente agradável/motivadora, requer feedback imediato, e deve ser realizada num curto período de tempo em cada dia, durante extensos períodos, sem levar à exaustão (Ericsson et al., 1993; Ericsson \& Lehmannn, 1996; Ericsson, Nandagopal, \& Roring, 2009; Ericsson \& Ward, 2007).

Da mesma forma são salientadas as competências perceptivo-cognitivas dos atletas com desempenhos excelentes e excepcionais. Alguns autores (ver Hodges, Huys, \& Starkes, 2007; Williams \& Ward, 2003) concluem pela superioridade dos experts em processos cognitivos, como a memória, percepção e antecipação, subjacentes aos "desempenhos superiores". Por último, investigações mais recentes (Connaughton, Wadey, Hanton, \& Jones, 2008; Duda \& Treasure, 2006; DurandBush \& Salmela, 2002; Gould, Dieffenbach, \& Moffet, 2002; Holt \& Dunn, 2004; Ruiz, Sánchez, Durán, \& Jiménez, 2006) destacam factores e processos psicológicos de natureza mais motivacional, afectiva e emocional. É o caso, entre outros, de elevados níveis de motivação, comprometimento e disciplina, concentração e focalização atencional, eficácia da liderança, auto-confiança e auto-conceito, competências de auto-regulação e regulação emocional, estratégias de visualização mental e de formulação de objectivos, resiliência/ resistência mental ou estratégias de planeamento e simulação. De algum modo, recuperam-se assim variáveis descritas no trabalho pioneiro de Griffith (1928) quando recorreu a termos como "luta", "esforço sobrehumano" ou "resistência mental" para descrever os atletas de sucesso.

\section{Competências psicológicas e sucesso desportivo}

O interesse pela compreensão da excelência impulsionou desde cedo, a investigação sobre as características e/ou competências dos atletas de sucesso, para além das estritamente físicas. Foi neste sentido que Morgan e colaboradores (Morgan, 1978, 1985; Morgan \& Costill, 1972) desenvolveram investigações iniciais na área, estudando as características de personalidade de atletas de elite e de nível mundial de diferentes modalidades, através do "Perfil de estados de Humor" (Profile of Mood States - POMS). Nestes estudos verificou-se que os atletas de sucesso evidenciavam um perfil psicológico do "tipo iceberg", com resultados inferiores à média da população em factores negativos como a tensão, depressão, raiva, fadiga e confusão, e resultados significativamente mais elevados no "vigorpsicológico", dimensões que integramos no conceito de "arquitectura" psicológica dos atletas de elite.

Numa perspectiva um pouco diferente, outros investigadores (Gould, Weiss, \& Weinberg, 1981; Mahoney \& Avener, 1977), procuraram avaliar e identificar estratégias ou competências psicológicas (e.g., autoverbalizações e visualização mental; estratégias de coping, formulação de objectivos, concentração, confiança, motivação para a realização), onde a "superioridade" dos atletas bem sucedidos se manifestava. Na continuidade desta lógica de investigação, Mahoney, Gabriel e Perkins (1987), numa amostra de 713 atletas de 23 desportos dos EUA, de diferentes níveis competitivos, identificaram as competências psicológicas relevantes para o rendimento e sucesso desportivo, utilizando o Inventário de Competências Psicológicas para o Desporto (Psychological Skills Inventory for Sports - PSIS). Os resultados demonstraram que os atletas de elite experienciavam menos problemas de ansiedade, evidenciavam maior concentração, auto-confiança, e motivação para o sucesso, recorriam mais à preparação mental, e centravam-se mais no seu próprio 
rendimento do que no da equipa. Na mesma altura, mas no Canadá, um outro estudo foi realizado por Orlick e Partington (1988) sobre as competências e "prontidão" mental de 235 atletas olímpicos, de 31 modalidades diferentes. Combinando metodologias qualitativas e quantitativas, os autores enfatizaram a utilização da visualização mental, o estabelecimento de objectivos, a simulação da competição, a elaboração de planos detalhados da competição e o desenvolvimento de planos para lidar com a distracção. Posteriormente, corroborando as conclusões de estudos anteriores, destacam-se igualmente as investigações de Gould e colaboradores (Gould, Eklund, \& Jackson, 1993; Gould et al., 1999; Greenleaf, Gould, \& Dieffenbach, 2001), onde foi também evidente a contribuição de outros factores mais contextuais (e.g., coesão de equipa, comunicação efectiva treinador-atleta e suporte positivo por parte dos outros significativos), na diferenciação e caracterização dos atletas de elite. Procurando colmatar uma lacuna da investigação e a focalização predominante no estudo de competências psicológicas durante a competição, Thomas, Murphy e Hardy (1999) desenvolveram para este efeito o Test of Performance Strategies (TOPS), avaliando a utilização das competências e estratégias psicológicas tanto no treino como na competição. Este instrumento, na sua primeira versão, foi aplicado junto de 472 atletas de diferentes níveis competitivos, permitindo concluir que as estratégias de motivação, visualização, relaxamento, controlo atencional e emocional eram fundamentais na preparação dos atletas para a competição, sendo igualmente utilizadas durante os treinos (Thomas et al., 1999).

Várias revisões da literatura e investigações posteriores (Jones, Hanton, \& Connaughton, 2002; Williams \& Krane, 2001) sintetizaram e especificaram as competências mentais e características psicológicas associadas a atletas olímpicos com elevados níveis de sucesso: (a) ter uma rotina e planos pré-competitivos e competitivos bem estruturados, (b) elevados níveis de motivação e comprometimento, concentração, auto-confiança e optimismo, (c) estratégias de coping eficazes para lidar com distracções e situações ou eventos inesperados, (d) regulação da activação e ansiedade, (e) estabelecimento e formulação de objectivos, e (f) visualização e prática mental. Por sua vez, a investigação nacional com atletas portugueses de elite (e.g., Barbosa \& Cruz, 1997; Bodas, Lázaro, \& Fernandes, 2007; Cruz, 1994, 1996; Dias, Cruz, \& Fonseca, 2009a, 2009b; Vasconcelos-Raposo, 1993) apresenta dados similares e evidencia padrões de competências e características comuns aos encontrados no "panorama" internacional. Mais recentemente, procurando também alguma sistematização dos factores que facilitam o sucesso dos atletas, MacNamara, Button e Collins (2010), realizaram um estudo qualitativo com 7 atletas de elite mundial e respectivos pais. Neste estudo, além de apresentarem uma síntese da investigação anterior sobre esta temática, os autores identificaram as características psicológicas para desenvolver a excelência (Psychological Characteristics of Developing Excellence - PCDEs), que incluíam a competitividade, o comprometimento, a percepção sobre o que é necessário para ser bem sucedido, a visualização, a importância de trabalhar os pontos fracos, o coping sob pressão, o conhecimento do jogo e a auto-confiança. De referir que algumas destas importantes dimensões psicológicas aparecem contempladas igualmente no "Athletic Coping Skills Inventory" (ACSI-28), desenvolvido por Smith, Schutz, Smoll e Ptacek (1995). Adicionalmente, sugerem que os programas de identificação e desenvolvimento do talento devem promover "precocemente" estas características psicológicas para optimizar o desenvolvimento e desempenho dos atletas.

Sintetizando, a investigação internacional e nacional em torno das características e competências dos atletas excepcionalmente bem sucedidos parece sugerir e evidenciar que o sucesso desportivo se associa ao ajustamento em termos psicológicos e à presença de um 
conjunto de características e competências importantes para lidar com a alta competição desportiva.

Mais direccionados para a compreensão da forma como se desenvolvem estas características psicológicas, podemos mencionar os trabalhos desenvolvidos por Bloom (1985), Côté (1999), Durand-Bush e Salmela (2002), Gould, Dieffenbach e Moffett (2002), Holt e Dunn (2004) e Morgan e Giacobbi (2006). Numa investigação qualitativa longitudinal, Bloom (1985) estudou um grupo de jovens talentosos, dos quais 21 eram nadadores olímpicos e 18 jogadores de ténis muito bem classificados em termos de ranking. O estudo identificou três estádios críticos no desenvolvimento do talento: os primeiros anos/estádio de iniciação (divertimento, actividades lúdicas, orientação, estimulação do interesse e apoio por parte dos pais), os anos intermédios/estádio de desenvolvimento (orientados para a realização, as crianças assumem as suas actividades de forma mais séria, praticam mais, e mostram mais dedicação para serem bem sucedidas), e os últimos anos/estádio da especialização e aperfeiçoamento (quando os indivíduos se tornam experts, mais autónomos e conhecedores sobre o seu treino e competição e a actividade em causa torna-se parte central na vida dos indivíduos). Posteriormente, Côté (1999) não só com o intuito de perceber o desenvolvimento da carreira desportiva mas também o papel da família nesse processo, levou a cabo um estudo qualitativo com 4 atletas de elite, das modalidades de remo e ténis, e respectivas famílias. A análise das entrevistas permitiu concluir a existência de três estádios de participação no desporto. Os "sampling years" (dos 6 aos 13 anos), que se caracterizam pela prática de uma grande variedade de actividades desportivas agradáveis e divertidas, incentivada pelos pais. Os "specializing years" (dos 13 aos 15 anos), por sua vez, correspondem àqueles em que os atletas se "especializam" numa ou duas das actividades em que se encontram envolvidos, procurando desenvolver competências específicas da modalidade. $\mathrm{O}$ apoio dos pais neste estádio faz-se sentir ao nível da ênfase dos desempenhos escolares e desportivos, do comprometimento em termos financeiros e de tempo e do interesse crescente pelo desporto do filho. Por último, nos "investment years" (por volta dos 15 anos), os atletas pretendem desenvolver-se ao nível da táctica e competências competitivas, daí que aumente o seu envolvimento em actividades de prática deliberada, procurando alcançar um nível elevado de excelência na modalidade. Os pais apoiam emocionalmente os atletas, ajudandoos a ultrapassar obstáculos. Partindo das limitações deste modelo, em que os estádios identificados não consideravam toda a carreira desportiva, Durand-Bush e Salmela (2002), num estudo com 10 campeões do mundo e campeões olímpicos concluíram a existência de quatro estádios, acrescentando aos propostos por Côté (1999) uma última etapa que denominaram de "anos de manutenção" (maintenence years), durante o qual os atletas continuam a treinar e competir, com o intuito de manterem e melhorarem $\mathrm{o}$ seu desempenho, sendo esta a fase em que habitualmente alcançam o topo da carreira desportiva. Neste estudo, os autores verificaram, ainda, que além de alguns atributos pessoais (paixão pelo desporto, confiança, trabalho árduo, perseverança, talento natural e determinação), a capacidade dos atletas se focarem no processo de desempenho, e não tanto nos resultados, também era um relevante factor para a manutenção do sucesso desportivo.

Reconhecendo a escassez de investigação relativa à forma como as competências psicológicas são cultivadas e desenvolvidas, Gould et al. (2002) estudaram as características psicológicas de 10 campeões olímpicos e os processos implicados no seu desenvolvimento desportivo. Em termos de resultados, identificaram algumas variáveis psicológicas centrais para a caracterização dos atletas excepcionais, nomeadamente: (a) auto- 
regulação da activação e ansiedade; (b) elevados níveis de auto-confiança, concentração e focalização atencional, determinação e compromisso, e controle emocional; (c) discurso interno e visualização mental de natureza positiva; (d) boas competências e recursos psicológicos de confronto com a competição desportiva; (e) planos mentais bem desenvolvidos; (f) formulação de objectivos e preparação mental; e (g) estratégias de controle do pensamento. Evidenciaram, ainda, algumas variáveis negligenciadas em pesquisas anteriores, como o perfeccionismo adaptativo, uma elevada orientação para o optimismo e para o "lado" positivo da vida, além de uma tendência disposicional para a esperança. Do mesmo modo, os resultados demonstraram a importância de diferentes pessoas e instituições (e.g., a comunidade, a família, as pessoas/ambiente do desporto), que podem influenciar o atleta de forma directa (e.g., ensinar ou enfatizar algumas "lições" psicológicas) e indirecta (e.g., modelagem ou criação/simulação de alguns ambientes psicológicos). Este estudo permitiu também confirmar os estádios identificados por Bloom (1985) e o papel crucial que as famílias desempenham no desenvolvimento dos talentos desportivos (e.g., Bloom, 1985; Coté, 1999).

Adicionalmente, Holt e Dunn (2004), no seu estudo qualitativo com 34 atletas de futebol e 6 treinadores, verificaram que uma carreira de sucesso no futebol profissional implica três etapas de desenvolvimento. Numa primeira etapa a criança tem de demonstrar competências acima da média. Na segunda, o atleta adolescente vai para uma organização profissional e, demonstrando as qualidades exigidas, mais facilmente passará à etapa seguinte em que se torna um jogador profissional de futebol. Num outro estudo qualitativo, em que participaram 8 atletas universitários de elevado sucesso, 12 pais e 6 treinadores, Morgan e Giacobbi (2006) concluíram que o desenvolvimento do talento resulta da interacção de um conjunto de factores de natureza genética, de treino, ambientais e psicológicos, que ocorre ao longo de 9 fases: (a) nascimento (aquisição de predisposições genéticas mentais e físicas); (b) iniciação no desporto (primeiras influências para participar no desporto por parte dos "outros significativos"); (c) desenvolvimento da juventude e influências (período de oportunidades ambientais e influências sociais críticas); (d) desenvolvimento das características psicológicas (e.g., motivação, estratégias de coping, e/ou competitividade); (e) decisão de se especializar (quando o atleta se encontra no secundário ou a transitar para a faculdade, sendo nesta altura que ocorre a especialização apenas numa modalidade); (f) "confrontar-se" com a adversidade (surge um acontecimento de vida crítico ou adverso); (g) lidar adequadamente com adversidades (aplica com sucesso as suas competências psicológicas e o sistema de suporte social prévio para ultrapassar esse obstáculo/adversidade); (h) consequência positiva (maior motivação para ser bem sucedido); e (i) resultado (torna-se num atleta de elevado sucesso, com oportunidades para progredir no desporto e na vida). Três aspectos merecem especial atenção neste estudo. Em primeiro lugar, refira-se que, face aos estudos anteriores, este é o único a salientar a importância dos factores genéticos no desenvolvimento do talento. Um segundo aspecto, reporta-se às fases identificadas e à sua "aproximação" aos acontecimentos de vida. De facto, enquanto os modelos anteriores estabelecem "marcos" desportivos como forma de diferenciar os estádios, este modelo faz predominantemente referência a situações e transições de vida que despoletam a passagem para um novo estádio (e.g., a entrada na faculdade). No entanto, saliente-se que, apesar deste estudo explorar o desenvolvimento do talento, as características da amostra (atletas universitários) tornam-na "qualitativamente inferior", quando comparada com as da investigação anterior, colocando assim alguns problemas de generalização dos resultados a atletas de elite. 
De um modo geral, os estudos abordados permitem apontar duas dificuldades centrais na investigação neste domínio. Por um lado, parece evidente que apesar da existência de alguns modelos de desenvolvimento de talento, ainda há um longo caminho a percorrer nesta área em face da escassez de investigações que se dediquem exclusivamente ao "seu" estudo no contexto desportivo, a que se associa alguma falta de consenso relativamente aos estádios a considerar. Por outro lado, parece ser evidente a existência de várias e diferentes trajectórias para a excelência. Como refere Durand-Bush e Salmela (2002) "os atletas podem seguir diferentes caminhos, usar vários recursos e estratégias, e serem inovadores e criativos à medida que desenvolvem e mantêm a sua expertise no desporto" (p. 169).

Concluindo, estes estudos, uns preocupados apenas com o que caracteriza os atletas de sucesso, outros interessados na compreensão da forma como estes desenvolvem o seu talento, demonstram a importância de uma grande variedade de características e competências psicológicas no desenvolvimento da excelência e sucesso desportivo. Entre estes factores, a relevância atribuída a variáveis e processos de natureza afectiva e emocional, bem como às estratégias de coping, parece ser evidente na investigação actual. Seguidamente explora-se com maior detalhe as relações destas variáveis com o sucesso desportivo, procurando sintetizar os principais dados evidenciados pela literatura neste domínio.

\section{Emoções, eficácia do coping e sucesso desportivo}

A investigação em torno dos estados emocionais no desporto centrou-se, inicialmente, sobretudo em emoções predominantemente negativas, como é o caso da ansiedade competitiva. Apenas nos últimos anos é que a Psicologia do Desporto começou a constatar que esta ênfase não contemplava outras reacções e experiências emocionais dos atletas (Gould et al., 2002; Hanin, 2000; Lazarus, 2000; Pensgaard \& Duda, 2003; Ruiz et al.,
2006), sugerindo a "variedade e a complexidade da vida emocional" em contextos desportivos (Cruz, 1996, p. 204). Na realidade, alguns estudos destacam que os atletas experienciam várias emoções de natureza positiva e negativa (ver Hanin, 2000; Lazarus, 2000), afirmando Skinner e Brewer (2004) que "perante tantas evidências de que as emoções positivas são comummente experienciadas pelos atletas durante as competições, é surpreendente que muito pouca investigação se tenha debruçado sobre as consequências destas emoções no desempenho actual" (p. 287). Estes autores defendem, por exemplo, a importância de se explorar as emoções de valência positiva experienciadas antes da competição e o seu impacto na preparação e desempenho dos atletas.

A par das emoções, também a forma como se lida com elas, ou seja, as estratégias e competências de coping têm sido estudadas e evidenciadas. Actualmente, já não existem dúvidas relativamente ao papel primordial desempenhado pelos processos de coping no sucesso e excelência desportiva (Gould et al., 1993, 1999), até porque os atletas com esse "estatuto" terão mesmo que ser capazes de lidar eficazmente com uma grande variedade de factores estressores e ambientes potencialmente ameaçadores para o seu bem-estar e auto-estima. Explicitando, Lazarus (2000) salienta que "o tipo adequado de coping numa competição importante pode levar os atletas a tornarem-se re-motivados e, consequentemente, capazes de manterem a atenção e concentração para desencadearem os seus padrões tipicamente elevados de excelência" (p. 237).

Quando abordamos o coping em contextos desportivos é impossível não ter em atenção os trabalhos de Lazarus e de Folkman que o descrevem como os esforços cognitivos, afectivos e comportamentais para lidar com exigências específicas externas ou internas, podendo ser de dois tipos: focado no problema ou focado nas emoções (Lazarus \& Folkman, 1984; Lazarus, 1991). O primeiro consiste nos 
esforços que visam actuar sobre a situação/problema e implica a utilização de estratégias que se centram na análise e definição do problema, criação de soluções, avaliação de custos e benefícios dessas soluções e a selecção da melhor solução. $\mathrm{O}$ segundo tipo de coping consiste nos esforços para regular as respostas emocionais resultantes de um estressor e implica a utilização de estratégias como a fuga/ evitamento, o distanciamento do problema, a atenção selectiva ou a desvalorização dos acontecimentos negativos (ver Cruz, 1996; Lazarus \& Folkman, 1984; Lazarus, 2000).

Várias têm sido as investigações em torno da identificação das competências de coping e da respectiva eficácia. Poczwardowski e Conroy (2002) procuraram identificar e categorizar as respostas de coping perante o sucesso e insucesso em oito atletas de elite de desportos individuais e colectivos e oito performers das artes (dança, ópera, música e teatro). Os autores concluíram que o reportório de estratégias de coping era variado e individual, e que os performers de elite usavam estas estratégias de forma combinada. $\mathrm{Na}$ mesma linha, uma investigação de Holt e Dunn (2004) sugere que: a) a avaliação e as respostas de coping interagem de forma transaccional e recursiva; b) os estressores são identificados quando os objectivos pessoais relevantes são colocados em causa; e c) o coping focado nas emoções parece ser a escolha mais adequada quando os estressores são incontroláveis, enquanto o coping focado no problema parece ser mais eficaz em situações controláveis, e o evitamento em resposta a estressores que são de curta duração e incontroláveis. Procurando perceber a natureza e significado do coping eficaz e adaptativo, Nicholls, Holt e Polman (2005) realizaram um estudo com atletas de elite no golfe, concluindo que a avaliação positiva, a paragem de pensamento e a adesão a rotinas pré-competitivas constituíam estratégias de coping eficazes. Numa outra investigação, mas com atletas internacionais de râguebi da Irlanda e Nova Zelândia (Nicholls,
Holt, Polman, \& Bloomfield, 2006), verificouse uma utilização diferenciada e combinada das diferentes estratégias de coping, sendo as mais utilizadas a concentração na tarefa, seguida da paragem de pensamento, do aumento de esforço e, por fim, da reavaliação positiva. Ainda a propósito da importância das competências de coping, refira-se um estudo de Pensgaard e Duda (2003), com 61 atletas olímpicos, demonstrando que o facto de os atletas experienciarem frequente-mente emoções positivas e "optimistas", resultava da eficácia do coping, sendo este o único preditor positivo do desempenho.

Finalmente, em Portugal, Dias et al. (2009a) estudaram 11 atletas portugueses de elite em várias modalidades desportivas quanto às (suas) principais fontes de estresse $\mathrm{e}$ ansiedade, estratégias de coping utilizadas e emoções experienciadas. A análise das entrevistas permitiu concluir que: (a) as principais fontes de estresse estavam relacionadas com aspectos ligados à natureza da competição, pressões externas e ao seu próprio desempenho; (b) os atletas recorriam simultaneamente a diversas estratégias de coping (centradas no problema e/ou nas emoções), por norma adaptativas; e, (c) além da ansiedade, existem outras emoções, positivas e negativas, que influenciam o seu desempenho.

Perante a importância atribuída aos factores afectivo-emocionais e ao processo de coping no rendimento e na excelência no desporto competitivo, importa conhecer como as emoções e reacções emocionais são activadas e geradas. Neste sentido, e sendo ainda difícil emergir uma teoria unificadora, a investigação recente tem sugerido crescente evidência para as vantagens teóricas e metodológicas de uma abordagem de natureza cognitiva, motivacional e relacional ao estudo do estresse, da ansiedade e de muitas outras emoções, positivas e negativas, num amplo "espectro emocional" que caracteriza os contextos desportivos (Cruz, 1994, 1996; Cruz \& Barbosa, 1998; Lazarus, 2000; Skinner \& Brewer, 2004). 


\section{CONCLUSÕES}

As contínuas exigências colocadas aos indivíduos e às instituições têm feito emergir uma preocupação, generalizada a diferentes domínios, com a compreensão da excelência e potencial humano. As questões inquietantes sobre este tópico relacionam-se, sobretudo, com aquilo que caracteriza os indivíduos excelentes, com a forma como estes desenvolveram essas características e com o modo como estes realizam as suas actividades.

A este respeito, verifica-se que, apesar de existir um grande interesse em torno do estudo e explicação da excelência, não surgiu ainda uma teoria unificadora e compreensiva. Esta dificuldade prende-se sobretudo com a complexidade inerente ao estudo da excelência, que se tem traduzido no aparecimento de diferentes modelos teóricos, entre os quais se salientam abordagens predominantemente associadas à sobredotação, expertise e sabedoria. Neste sentido, a análise das teorias e estudos da excelência em contextos desportivos permite-nos verificar que este fenómeno tem sido associado a variáveis de carácter social e contextual, à prática deliberada, às competências perceptivocognitivas e a variáveis psicológicas de natureza motivacional, afectiva e emocional. Recentemente, Baker e Horton (2004), numa revisão sobre a aquisição e manifestação de desempenhos excepcionais, sugerem a organização dos factores em dois tipos: os primários e os secundários. Os factores primários têm uma influência directa na aquisição de desempenhos excepcionais e incluem os elementos de carácter pessoal, onde se inserem os factores de treino e prática deliberada, os factores perceptivo-cognitivos e os factores motivacionais, afectivos e emocionais. Os factores secundários influenciam frequentemente os primários e dizem respeito aos aspectos sociais e contextuais, como o suporte social ou o apoio de "outros significativos".

No presente estudo, entre os factores de influência primária demos especial ênfase às características e competências psicológicas e às dimensões afectivas e emocionais que, segundo alguns autores (e. g., Cruz, 1994, 1996; Dias et al., 2009a, 2009b; Gould et al., 2002; Pensgaard \& Duda, 2003), assumem um papel crucial na compreensão do desenvolvimento e manutenção de desempenhos de alto nível no desporto. De acordo com a nossa síntese, a excelência desportiva associa-se a níveis elevados de motivação e comprometimento, ao uso de estratégias de coping adaptativas, a elevados níveis de concentração e de autoconfiança, à auto-regulação, à formulação de objectivos, e às estratégias de visualização. Este "perfil" psicológico que caracteriza os "melhores" vai sendo construído e desenvolvido com o tempo, sendo influenciado por um conjunto de pessoas significativas para os atletas (e.g., pais, treinadores, dirigentes, colegas) e por diferentes organizações e instituições desportivas.

Considerando a importância das dimensões afectivas e emocionais, a capacidade do atleta para se confrontar com uma determinada situação, utilizando, para isso, estratégias de coping eficazes, parece ter um papel determinante no seu sucesso. Aliás, são cada vez mais frequentes as investigações centradas no papel das emoções e no estudo da eficácia do coping em termos de rendimento e sucesso competitivo. Esta associação é assinalada por Poczwardowski e Conroy (2002) ao defenderem que "a excelência no coping precede a excelência no desempenho" (p. 313).

Assim, de um modo quase consensual, a investigação considera que as competências específicas no domínio e as qualidades físicas deixaram de constituir um "passaporte para o êxito", passando outras variáveis a assumir um papel de destaque. Neste sentido, torna-se fundamental o estudo das variáveis psicológicas que influenciam $o$ rendimento desportivo, tendo em vista a compreensão de uma potencial "arquitectura" psicológica dos atletas excelentes. Traçar este "perfil de competências" ou desvendar uma tal "arquitectura" psicológica não parece consti- 
tuir tarefa fácil, uma vez que a excelência está associada a diferentes dimensões, não se sabendo contudo o peso ou a importância de cada uma delas e, em menor grau, de que forma é que se combinam. Esperamos que a presente síntese, em torno dos atletas com desempenhos excepcionais, possa incentivar uma nova "vaga" de investigação tendo em vista a compreensão do "fenómeno" da excelência no desporto.

\section{REFERÊNCIAS}

Almeida, L. S., \& Oliveira, E. P. (2000). Os professores na identificação dos alunos sobredotados. In L. S. Almeida, E. P. Oliveira, \& A. S. Melo (Eds.), Alunos sobredotados: Contributos para a sua identificação e apoio (pp. 4353). Braga: ANEIS.

Araújo, L. S., Cruz, J. F., \& Almeida, L. S. (2007). Excelência humana: Teorias explicativas e papel determinante do professor. Psicologia, Educação e Cultura, XI, 197-221.

Araújo, L. S., Cruz, J. F., \& Almeida, L. S. (2009). Pathways of excellence in science and dance: Lessons learned from a Portuguese case study. In A. Williamon, S. Pretty, \& R. Buck (Eds.), Proceedings of the International Symposium on Performance Science (pp. 139-144). Utrecht, The Netherlands: EAC.

Baker, J., \& Horton, S. (2004). A review of primary and secondary influences on sport expertise. High Ability Studies, 15, 211-228.

Baltes, P. B. (1993). The aging mind: Potential and limits. Gerontologist, 33, 580-594.

Baltes, P., \& Kunzmann, U. (2004). The two faces of wisdom: Wisdom as a general theory of knowledge and judgment about excellence in mind and virtue vs. wisdom as everyday realization in people and products. Human Development, 47, 290-299.

Baltes, P., \& Staudinger, U. (2000). A metaheuristic (pragmatic) to orchestrate mind and virtue toward excellence. American Psychologist, 55, 122-136.

Barbosa, L. G., \& Cruz, J. F. (1997). Estudo do stress, da ansiedade e das estratégias de confronto psicológico no andebol de alta competição. Psicologia: Teoria, Investigação e Prática, 2, 523-548.

Bloom, B. S. (1985). Developing talent in young people. New York: Ballantine.
Bodas, A., Lázaro, J, \& Fernandes, H. (2007). Perfil psicológico de prestação dos atletas paralímpicos Atenas 2004. Motricidade, 3(3), 33-43.

Connaughton, D., Wadey, R., Hanton, S., \& Jones, G. (2008). The development and maintenance of mental toughness: Perceptions of elite performers. Journal of Sports Sciences, 26, 83-95.

Côté, J. (1999). The influence of the family in the development of talent in sport. The Sport Psychologist, 13, 395-417.

Cruz, J. F. (1994). Stress, ansiedade e rendimento na competição desportiva: A importância das competências e processos psicológicos (Dissertação de doutoramento não publicada). Instituto de Educação e Psicologia, Universidade do Minho, Portugal.

Cruz, J. F. (1996). Stress, ansiedade e rendimento na competição desportiva. Braga: Edições do Centro de Estudos em Educação e Psicologia, Universidade do Minho.

Cruz, J. F., \& Barbosa, L. G. (1998). Stress, ansiedade e confronto psicológico na competição desportiva: Uma nova abordagem de natureza cognitiva, motivacional e relacional. Psicologia: Teoria, Investigação e Prática, 3, 21-70.

De Groot, A. D. (1978). Thought and choice in chess. The Hague: Mouton.

Dias, C., Cruz, J. F., \& Fonseca, A. F. (2009a). Emoções, stress, ansiedade e coping: Estudo qualitativo com atletas de elite. Revista Portuguesa de Ciências do Desporto, 9, 9-23.

Dias, C., Cruz, J. F., \& Fonseca, A. F. (2009b). Anxiety and coping strategies in sport context: A look at the psychometric properties of the Portuguese instruments for their assessment. The Spanish Journal of Psychology, 12, 338-348.

Duda, J. L., \& Treasure, D. C. (2006). Toward optimal motivation in sport: Fostering athletes competence and sense of control. In J. Williams (Ed.), Applied sport psychology: Personal growth to peak performance ( $5^{\mathrm{a}}$ ed., pp. 57-82). New York: McGraw-Hill.

Durand-Bush, N., \& Salmela, J. H. (2002). The development and maintenance of expert athletic performance: Perceptions of World and Olympic champions. Journal of Applied Sport Psychology, 14, 154-171.

Ericsson, K. A. (Ed.) (1996). The road to excellence: The acquisition of expert performance in the arts and 
sciences, sports and games. Hillsdale, NJ: Lawrence Erlbaum Associates.

Ericsson, K. A., \& Charness, N. (1994). Expert performance: Its structure and acquisition. American Psychologist, 49, 725-747.

Ericsson, K. A., \& Lehmannn, A. C. (1996). Expert and exceptional performance: Evidence of maximal adaptation to task constraints. Annual Review of Psychology, 47, 273-305.

Ericsson, K. A., \& Ward, P. (2007). Capturing the naturally occurring superior performance of experts in the laboratory: Toward a science of expert and exceptional performance. Current Directions in Psychological Science, 16, 346-350.

Ericsson, K. A., Krampe, R. T., \& Tesch-Römer, C. (1993). The role of deliberate practice in the acquisition of expert performance. Psychological Review, 100, 363-406.

Ericsson, K. A., Nandagopal, K., \& Roring, R. W. (2009). Toward a science of exceptional achievement: Attaining superior performance through deliberate practice. Annals of the New York Academy of Sciences, 1172, 199-217.

Gagné, F. (2004). Transforming gifts into talents: The DMGT as a developmental theory. High Ability Studies, 15, 119-147.

Gagné, F. (2007). Ten commandments for academic talent development. Gifted Child Quarterly, 51, 93-118.

Galton, F. (1869). Hereditary genius: An inquiry into its laws and consequences. London: Macmillan.

Garcia-Santos, S. C., Almeida, L. S., Werlang, B. S. G., \& Veloso, A. L. M. (2010). Processamento da informação em gestores de alto desempenho. Motricidade, 6(1), 85-102.

Gould, D., Dienffenbach, K., \& Moffett, A. (2002). Psychological characteristic and their development in Olympic champions. Journal of Applied Sport Psychology, 14, 172-204.

Gould, D., Eklund, R. C., \& Jackson, S. (1993). Coping strategies used by more versus less successful Olympic wrestlers. Research Quarterly for Exercise and Sport, 64, 83-93.

Gould, D., Guinan, D., Greenleaf, C., Medbery, R., \& Peterson, K. (1999). Factors affecting Olympic performance: Perceptions of athletes and coaches from more and less successful teams. The Sport Psychologist, 13, 371-394.

Gould, D., Weiss, M., \& Weinberg, R. (1981). Psychological characteristics of successful and nonsuccessful big ten wrestlers. Journal of Sport Psychology, 3, 69-81.
Greenleaf, C. A., Gould, D., \& Dieffenbach, K. (2001). Factors influencing Olympic performance: Interviews with Atlanta and Nagano U.S. Olympians. Journal of Applied Sport Psychology, 13, 179-209.

Griffith, C. R. (1928). Psychology and athletics. New York: Scribners.

Hanin, Y. L. (2000). Introduction: An individualized approach to emotion in sport. In Y. L. Hanin (Ed.), Emotions in sport (pp. ix-xii). Champaign, IL: Human Kinetics.

Hodges, N., Huys, R., \& Starkes, J. (2007). Methodological review and evaluation of research in expert performance in sport. In G. Tenenbaum, \& R.C. Eklund (Eds.), Handbook of sport psycho$\log y$ (pp. 161-183). Hoboken, NJ: John Wiley.

Holt, N. L. \& Dunn, J. (2004). Toward a grounded theory of the psychosocial competencies and environmental conditions associated with soccer success. Journal of Applied Sport Psychology, 16, 199-219.

Jones, G. D., Hanton, S., \& Connaughton, D. (2002). What is this thing called mental toughness? An investigation of elite sport performers. Journal of Applied Sport Psychology, 14, 205-218.

Kunzmann, U., \& Baltes, P. B. (2003). Wisdomrelated knowledge: Affective, motivational, and interpersonal correlates. Personality and Social Psychology Bulletin, 29, 1104-1119.

Kunzmann, U., \& Baltes, P. B. (2005). The psychology of wisdom: Theoretical and empirical challenges. In R. J. Sternberg, \& J. Jordan (Eds.), Handbook of wisdom: Psychological perspectives (pp. 110-135). Cambridge: University Press.

Lazarus, R. S. (1991). Emotion and adaptation. New York: Oxford University Press.

Lazarus, R. S. (2000). How emotions influence performance in competitive sports. The Sport Psychologist, 14, 229-252.

Lazarus, R. S., \& Folkman, S. (1984). Stress, appraisal and coping. New York: Springer.

MacNamara, A., Button, A., \& Collins, D. (2010). The role of psychological characteristics in facilitating the pathway to elite performance part 1: Identifying mental skills and behaviors. The Sport Psychologist, 24, 52-73.

Mahoney, M. J, \& Avener, M. (1977). Psychology of the elite athlete: An exploratory study. Cognitive Therapy and Research, 1, 135-141. 
Mahoney, M. J., Gabriel, T. J., \& Perkins, R. S. (1987). Psychological skills and exceptional athletic performance. The Sport Psychologist, 1, 135-141.

Monteiro, S., Castro, M., Almeida, L. S., \& Cruz, J. F. A. (2009). Alunos de excelência no ensino superior: Comunalidades e singularidades na trajectória académica. Análise Psicológica, 27(1), 79-87.

Morgan, T. K., \& Giacobbi, P. R., (2006). Toward two grounded theories of the talent development and social support process of highly successful collegiate athletes. The Sport Psychologist, 20, 295-313.

Morgan, W. P. \& Costill, D. L. (1972). Psychological characteristics of the marathon runner. Journal of Sports Medicine, 12, 42-46.

Morgan, W. P. (1978). The mind of the marathoner. Psychology Today, 11, 38-49.

Morgan, W. P. (1985). Selected psychological factors limiting performance: A mental health model. In D. H. Clarke, \& H. M. Eckert (Eds.), Limits of human performance (pp. 70-81). Champaign, II: Human Kinetics.

Nicholls, A., Holt, N., \& Polman, R. (2005). A phenomenological analysis of coping effectiveness in golf. The Sport Psychologist, 19, 111130.

Nicholls, A., Holt, N., Polman R., \& Bloomfield, J. (2006). Stressors, coping, and coping effectiveness among professional rugby union players. The Sport Psychologist, 20, 314-329.

Orlick, T., \& Partington. J. (1988). Mental links to excellence. The Sport Psychologist, 2, 105-130.

Pensgaard, A. M., \& Duda, J. L. (2003). Sidney 2000: The interplay between emotions, coping, and the performance of Olympic-level athletes. The Sport Psychologist, 17, 253-267.

Poczwardowski, A., \& Conroy, D. E. (2002). Coping responses to failure and success among elite athletes and performing artists. Journal of Applied Sport Psychology, 14, 313-329.

Ruiz, L., Sánchez, M., Durán, J., \& Jiménez, C. (2006). Los expertos en el deporte: Su estudio y análisis desde una perspectiva psicológica. Anales de Psicologia, 22, 132-142.

Salas, E., Cannon-Bowers, J. A., \& Johnston, J. H. (1997). How can you turn a team of experts into an expert team? Emerging training strategies. In C. Zsambok, \& G. Klein (Eds.), Naturalistic decision making: Where are we now? (pp. 359-370). Hillsdale: Lawrence Erlbaum.
Simon, H. A., \& Chase, W. G. (1973). Skill in chess. American Scientist, 61, 394-403.

Skinner, N., \& Brewer, N. (2004). Adaptative approaches to competition: Challenge appraisals and positive emotion. Journal of Sport E Exercise Psychology, 26, 283-305.

Smith, R. E., Schutz, R., Smoll, F. L., \& Ptacek, J. T. (1995). Development and validation of a multidimensional measure of sport-specific psychological skills: The Athletic Coping Skills Inventory-28. Journal of Sport \& Exercise Psychology, 17, 379-398.

Staudinger, U. M., Maciel, A. G., Smith, J., \& Baltes, P. (1998). What predicts wisdom-related performance? A first look at personality, intelligence, and facilitative experiential contexts. European Journal of Personality, 12, 1-17.

Sternberg, R. J. (1998). Metacognition, abilities, and developing expertise: What makes an expert student? Instructional Science, 26, 127-140.

Sternberg, R. J. (2001). Giftedness as developing expertise: A theory of the interface between high abilities and achieved excellence. High Ability Studies, 12, 159-179.

Sternberg, R. J. (2005). WICS: A model of leadership. The Psychologist - Manager Journal, 8, 29-43.

Terman, L. M. (1925). Genetic Studies of genius (Vol. 1): Mental and physical traits of a thousand gifted children. Stanford: Stanford University Press.

Thomas, K., \& Thomas, J. (1994). Developing expertise in sport: The relation of knowledge and performance. International Journal of Sport Psychology, 25, 295-312.

Thomas, P. R., Murphy, M. S., \& Hardy, L. (1999). Test of performance strategies: Development and preliminary validation of a comprehensive measure of athletes psychological skills. Journal of Sports Sciences, 17, 697-711.

Vasconcelos-Raposo, J. V. (1993). Os factores psicosocio-culturais que influenciam e determinam a busca da excelência pelos atletas de elite desportiva portuguesa (Dissertação de doutoramento não publicada). Universidade de Trás-os-Montes e Alto Douro, Vila Real, Portugal.

Ward, P., Hodges, N., Starkes, J., \& Williams, M. (2007). The road to excellence: Deliberate practice and the development of expertise. High Ability Studies, 18, 119-153.

Williams, A., \& Ericsson K. (2005). Perceptualcognitive expertise in sport: Some considerations when applying the expert performance approach. Human Movement Science, 24, 283-307. 
Williams, A., \& Ward, P. (2003). Perceptual expertise: Development in sport. In J. Starkes, \& K. A. Ericsson (Eds.), Expert performance in sport: Advances in research on sport expertise (pp. 219-250). Champaign, Ill.: Human Kinetics.
Williams, J. M., \& Krane, V. (2001). Psychological characteristics of peak performance. In J. M. Williams (Ed.), Applied sport psychology: Personal growth to peak performance (4th ed., pp. 137147). Mountain View, CA: Mayfield.

(cc) EY-No Todo o conteúdo da revista Motricidade está licenciado sob a Creative Commons, excepto quando especificado em contrário e nos conteúdos retirados de outras fontes bibliográficas. 